\title{
Endobronchial variant of sclerosing hemangioma of the lung: histological and cytological features on endobronchial material
}

\author{
Mojgan Devouassoux-Shisheboran ${ }^{1}$, Arnaud de la Fouchardière ${ }^{1}$, Françoise Thivolet- \\ Béjui $^{1}$, Marie L Sourisseau-Millan ${ }^{1}$, Jean C Guerin ${ }^{2}$ and William D Travis ${ }^{3}$ \\ ${ }^{1}$ Department of Surgical Pathology; ${ }^{2}$ Department of Respiratory Disease, Hôpital de la Croix Rousse, Lyon, \\ Cedex 04, France and ${ }^{3}$ AFIP, Department of Lung and Mediastinal Pathology, Washington, DC, USA
}

\begin{abstract}
We report two cases of a rare presentation of pulmonary sclerosing hemangioma as an endobronchial polyp resulting in clinical symptoms of bronchial obstruction. We describe the histological and cytological description of this entity based on material obtained during bronchoscopic examination. In both cases, bronchoscopy revealed a polypoid mass protruding into the left lower lobar bronchus, with a smooth and hemorrhagic surface, reminiscent of a carcinoid tumor. On bronchial washing in case 1, numerous small round cells were seen with uniform nuclei and inconspicuous nucleoli, arranged in clusters with a pavement-type pattern and papillary configuration. The presence of a few reactive pneumocytes gives a dual cell proliferation, in a background of numerous foamy macrophages. Although the four architectural patterns of sclerosing hemangioma were present in the resected tumors, the papillary and solid patterns predominated in the endobronchial component of the tumors and these were seen on the bronchial biopsies. Recognition of the papillary pattern, the typical round cells coexpressing thyroid transcription factor- 1 and epithelial membrane antigen without cytokeratin immunoreactivity, are helpful clues for the diagnosis on bronchial biopsy. Finally, hopefully awareness by clinicians and pathologists that sclerosing hemangioma can present as an endobronchial mass will facilitate diagnosis when this rare event occurs.

Modern Pathology (2004) 17, 252-257, advance online publication, 2 January 2004; doi:10.1038/modpathol.3800045
\end{abstract}

Keywords: sclerosing hemangioma; lung; bronchial washing; cytology; endobronchial; bronchial biopsy

Sclerosing hemangioma is a rare pulmonary neoplasm deriving from primitive respiratory epithelium. ${ }^{1}$ In almost all cases, the clinical presentation is that of a well-circumscribed peripheral or subpleural nodule, incidentally found on a chest X-ray. ${ }^{2}$ Given the peripheral location of the tumor, bronchoscopic examination typically does not reveal any abnormality. Preoperative pathologic material usually consists of fine-needle aspiration (FNA) biopsy. Endobronchial extension of sclerosing hemangioma is extremely rare, representing $1 \%$ of reported cases. ${ }^{1}$ The histological and cytological features of sclerosing hemangioma on endobronchial material such as bronchial biopsy and bronchial washing have never been reported. We report a rare, little known presentation of pulmonary sclerosing hemangioma, discovered as an endobronchial polyp, with details on the clinical presentation,

Correspondence: M Devouassoux-Shisheboran, Service d'Anatomie Pathologique, Hôpital de la Croix Rousse, 93, Grande Rue de la Croix Rousse, 69317, Lyon, Cedex 04, France.

E-mail: mojgan.devouassoux-shisheboran@chu-lyon.fr

Received 14 May 2003; revised 22 August 2003; accepted 25 August 2003; published online 2 January 2004 histological and cytological features on endobronchial biopsy material.

\section{Material and methods}

We reviewed two cases of sclerosing hemangioma of the lung presented as an endobronchial polyp identified between 1980 and 2001 in the files of Croix Rousse hospital. Cases without endobronchial component were excluded from the study.

\section{Cytological Material}

The bronchial washings were centrifuged at a rates of 1500 rates per minute for $20 \mathrm{~min}$. The sediment obtained was smeared on five slides, promptly fixed in $95 \%$ ethanol and stained with Papanicolaou stain (EA 65).

\section{Histological Material}

The bronchial biopsies (five fragments in case 1 and two fragments in case 2 , of $1 \mathrm{~mm}$ each) were 
formalin-fixed and paraffin-embedded. The left lower lobectomies were formalin-fixed and representative tissue blocks of the tumors (eight in case 1 and six in case 2) were sampled. Sections, $4 \mu \mathrm{m}$ thick, were cut and stained with hematoxylin, eosin and safron. Immunohistochemical study for cytokeratin (AE1/AE3, Dako, Glostrup, Denmark, working dilution: 1/50), EMA (epithelial membrane antigen, Dako, 1/100), cytokeratin 5/6 (Dako, 1/50), chromogranin (Dako, 1/150), synaptophysin (Dako, 1/50), thyroglobulin (Dako, 1/50) and TTF-1 (thyroid transcription factor 1, Zymed, San Francisco, CA, USA, 1/50) was performed on tissue sections, using streptavidin-biotin-peroxidase complex technique.

\section{Results}

\section{Clinical Features}

The patients were nonsmoker female subjects, aged 44 and 51 years (mean age 47 years). Both were symptomatic presenting in the first case with dyspnea and cough 10 months after a subtotal thyroidectomy for papillary carcinoma. The second patient presented with recurrent acute bronchitis and pneumonia with mucinous secretion over a period of 8 months. In the first case, the chest X-ray showed a left hilar opacity, and the CT-scan demonstrated a 5-cm well-circumscribed and homogenous nodule, without calcification. Chest X-ray and CT-scan did not show any abnormality in the second patient. In both cases, bronchoscopic examination revealed a polypoid tumor within the lumen of the left lower lobar bronchus. The tumors had a smooth and hemorrhagic external surface, and measured 5 and $10 \mathrm{~mm}$ in diameter, respectively. In both cases, the bronchoscopic impression was probable carcinoid tumor. Multiple biopsies and bronchial washing of the endobronchial masses were performed. Both patients were referred to our institution for lung surgery and they underwent left lower lobectomy. Both are well without evidence of disease 3 and 2 years after the diagnosis, respectively.

\section{Bronchial Washing Cytology Findings}

In the first case, the smears of the bronchial washings were moderately cellular. The background was slightly hemorrhagic, without necrosis. In addition to the cellular components usually seen on bronchial cytology (bronchial ciliated columnar cells, alveolar macrophages, squamous cells from salivary contamination), numerous clusters and sheets of mononuclear cells were found. These cells were small to medium in size, bland looking and without mitoses. They had round to oval nuclei, evenly dispersed chromatin, inconspicuous nucleoli and very scant cyanophilic cytoplasm (Figure 1). Some cells tended to cluster, assuming a papillary configuration (Figure 2). Other clusters displayed a

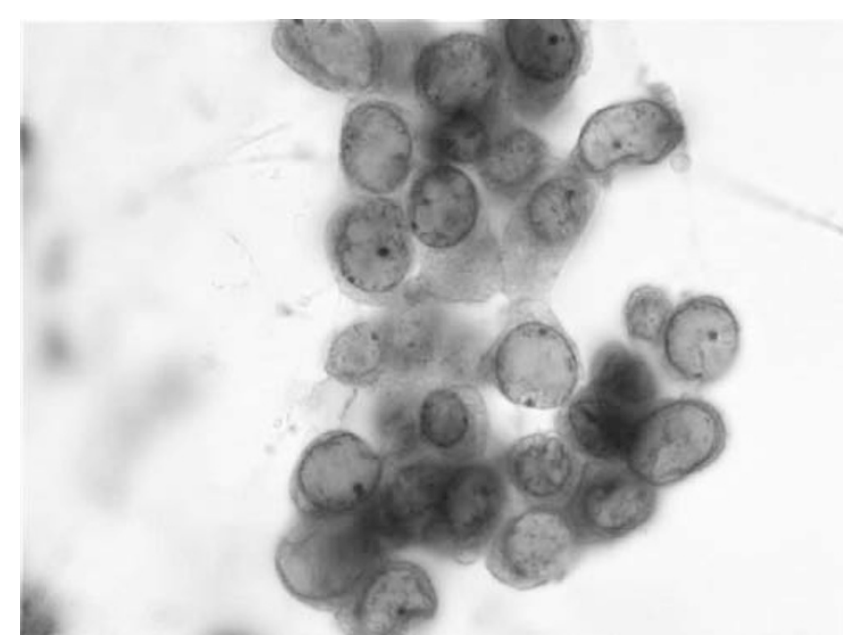

Figure 1 Bronchial washing case 1: round tumor cells are small, round with scant cyanophilic cytoplasm, uniform nuclei and inconspicuous nucleoli (Papanicolaou $\times 1000)$.

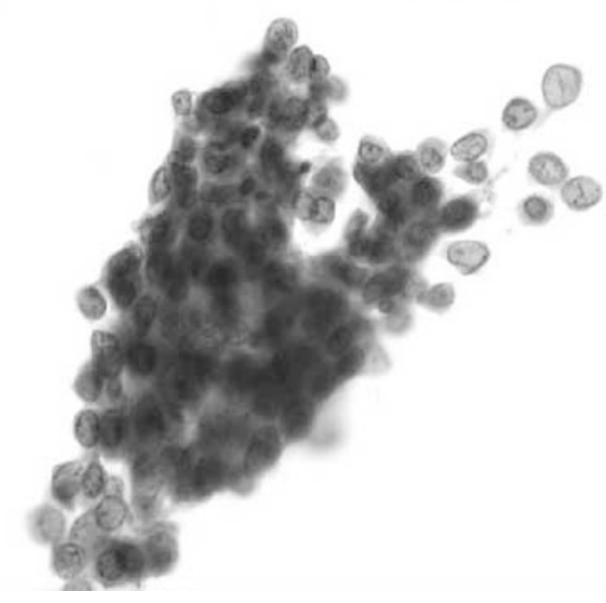

Figure 2 Bronchial washing case 1: round tumor cells clustering in a papillary configuration. A few nuclei have irregular and grooved membrane (Papanicolaou $\times 400$ ).

pavement-type pattern, composed of round to polygonal cells with centrally located nuclei and welldefined cytoplasmic borders (Figure 3). A few isolated cells were also seen, sometimes in the form of naked nuclei. In addition to these small cells, more epithelial-looking cells reminiscent of pneumocytes were focally present. They were larger, and had an abundant cyanophilic cytoplasm and welldefined cell borders. Numerous foamy macrophages were also seen.

In the second case, bronchial washing did not demonstrate any tumor cells.

\section{Bronchial Biopsy Findings}

The histologic examination of the endobronchial biopsies in both cases revealed a papillary and solid proliferation, with ulceration of the normal 


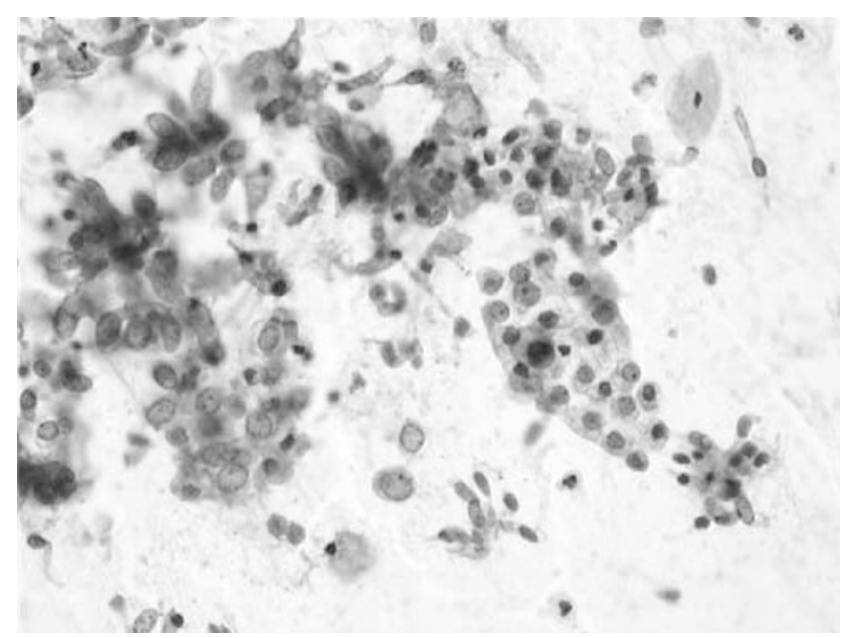

Figure 3 Bronchial washing case 1: small, round cells arranged in a pavement-type pattern in a background of foamy macrophages and respiratory ciliated cells (Papanicolaou $\times 200$ ).

bronchial mucosa. The papillary architecture predominated in both cases. The papillae were centered by a vessel displaying a thick hyalinized and sometimes calcified wall, and were covered by sheets of small cells (Figures 4 and 5). These cells had the typical aspects of round cells, described in sclerosing hemangioma of the lung. ${ }^{2}$ They were uniform and bland-appearing and contained round to oval nuclei with fine chromatin and inconspicuous nucleoli. They contained abundant eosinophilic cytoplasm. A few hyperchromatic, slightly enlarged nuclei were also seen. However, mitotic figures were absent. The papillae were dissociated from each other, and a few small cells were detached from the cluster. In addition, a few larger, alveolar cells were observed. They were covering some of the papillae, or were admixed within clusters of small cells, forming acinar structures. They usually had a foamy and clarified cytoplasm and small nuclei without atypia or mitosis. In case 1 , the residual bronchial mucosa was covering some of the fragments and was composed of ciliated and columnar cells, undergoing focally squamous metaplasia.

The immunohistochemical study of both cases revealed a membranous EMA staining and a TTF-1 nuclear staining of both cell types (small cells and alveolar cells), while only alveolar epithelium was positively immunostained with anti-cytokeratin antibody. Neuroendocrine markers were negative. Given the prior history of papillary thyroid carcinoma in the first patient, a thyroglobulin stain was negative.

\section{Left Lower Lobectomy Specimens}

In the first case, a large 5 -cm solid, well-circumscribed nodule was seen. The nodule was in contact with the mediastinal pleura and had an endobronchial component, in the form of a $5 \mathrm{~mm}$ polypoid mass protruding into the bronchial lumen.

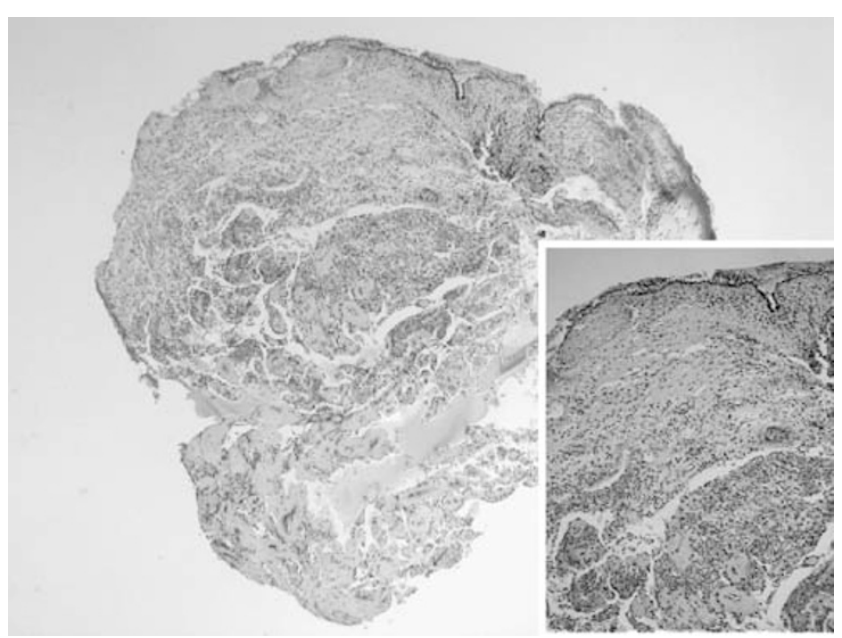

Figure 4 Bronchial biopsy in patient 1: papillary and solid proliferation overlined by respiratory epithelium $($ HPS $\times 25$ ); inset (HPS $\times 100)$.

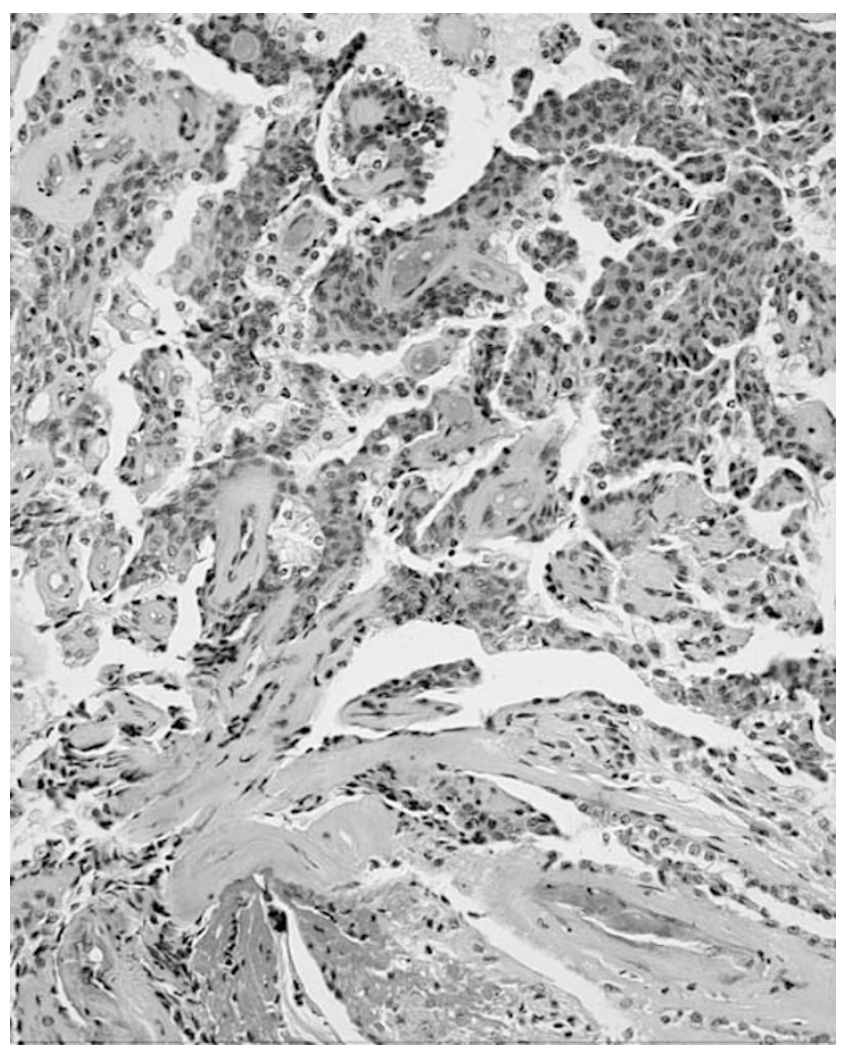

Figure 5 Bronchial biopsy in patient 1: papillary proliferation composed of small cells with uniform nuclei and alveolar cells with a clarified cytoplasm, surrounding a papillary core characterized by a fibrous, thick wall vessel $($ HPS $\times 200)$.

In the second case, a polypoid mass of $2 \times 0.5 \mathrm{~cm}$ in diameter was protruding into the left lower bronchial lumen (Figure 6). This mass extended into the lung parenchyma, forming a $1 \mathrm{~cm}$, solid, well-circumscribed nodule.

Histologically, both cases showed all four patterns described in sclerosing hemangiomas ${ }^{2}$ with papillary, 


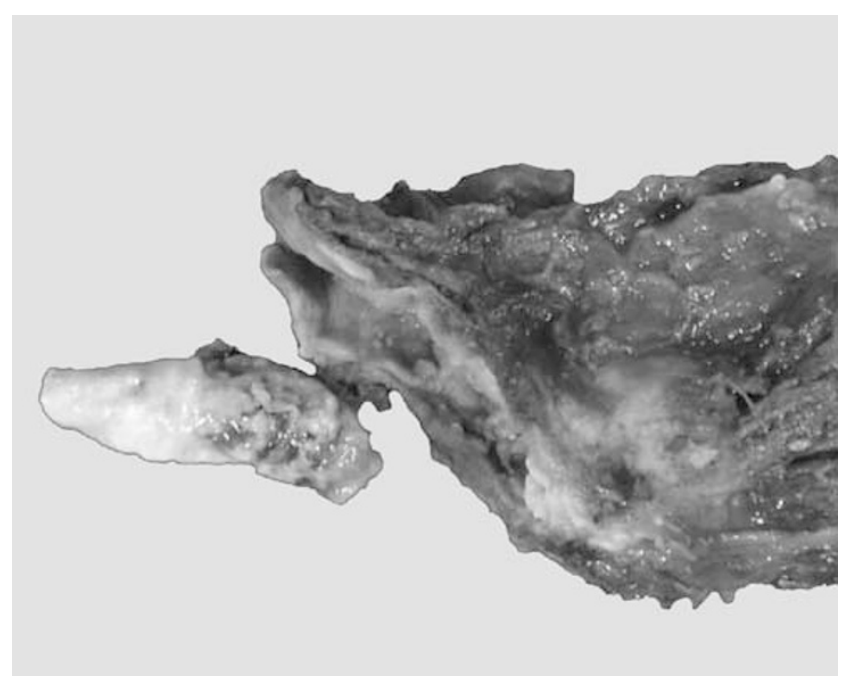

Figure 6 Gross examination of the left lower lobectomy in patient 2: the bulk of the lesion was endobronchial presenting as a $2.5 \times 0.5 \mathrm{~cm}$ polypoid mass protruding into the bronchial lumen.

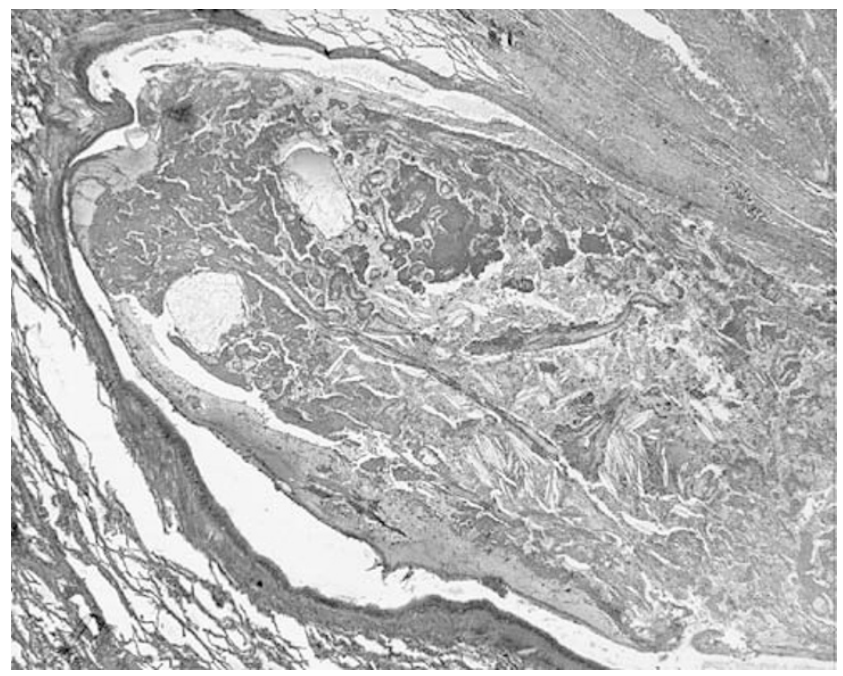

Figure 7 Left lower lobectomy case 1: sclerosing hemangioma protruding into the bronchial lumen (HPS $\times 20$ ).

solid, sclerosing and hemorrhagic areas. The tumor invaded and destroyed the bronchial wall, protruding as a polypoid mass into the bronchial lumen (Figure 7).

The immunohistochemical staining results were identical to that of the biopsy specimens with a coexpression of TTF-1 and EMA in both round and alveolar cells and pancytokeratin expression in the alveolar cells only. Neither round nor alveolar cells expressed cytokeratin 5/6.

\section{Discussion}

Although sclerosing hemangioma is a rare neoplasm in the lung, its clinical, radiological and histological features are well documented. We present here two cases of pulmonary sclerosing hemangioma with an unusual clinical presentation. Both our cases were initially discovered as an endobronchial polypoid mass on bronchoscopy that caused diagnostic difficulties to both clinicians and pathologists. Indeed, our patients underwent bronchoscopy with bronchial washing and biopsy. In both cases the clinical diagnosis was that of a carcinoid tumor. Also, in none of the cases, was the correct diagnosis of sclerosing hemangioma rendered by pathologists at outside institutions who initially examined the endobronchial material. The patients were referred to our institution with a diagnosis of 'probable papillary thyroid carcinoma, metastasis' for the first patient and 'papillary adenoma' for the second patient. The possibility of an endobronchial presentation of sclerosing hemangioma was not considered by either the clinicians or pathologists initially involved with these cases.

An endobronchial spread of sclerosing hemangioma is rare, occurring in only $1 \%$ of cases in one large series. ${ }^{1}$ However, in that case, the tumor presented initially as a peripheral nodule and the endobronchial extension into a medium-sized, segmental, peripheral bronchus was seen only after histological examination of the resected peripheral tumor. Our cases are the first description of sclerosing hemangioma presenting initially as an endobronchial polypoid mass, into a main bronchus.

Both our patients were nonsmoking female subjects aged 44 and 51 years (mean age 47 years). This is consistent with the known epidemiological aspects of peripheral sclerosing hemangioma, occurring predominately in middle-aged females. ${ }^{2}$ However, the endobronchial extension is responsible for a peculiar clinical and radiological presentation of sclerosing hemangioma. Although $80 \%$ of peripheral sclerosing hemangioma are asymptomatic, and discovered incidentally on a routine chest X-ray, ${ }^{1,2}$ both our patients were symptomatic presenting with 8 and 10 months of clinical signs of bronchial obstruction, such as dyspnea, cough and iterative acute bronchitis. These clinical signs were due to the presence of a mass obstructing the main left lower lobar bronchus in both cases. Interestingly, both our cases occurred in the left lower lobar bronchus, while peripheral sclerosing hemangioma have been reported in all segments of lung parenchyma. ${ }^{1,2}$ Peripheral sclerosing hemangioma presents as a coin lesion that is obvious on chest X-ray, thoracic CT-scan and MRI. However, no peripheral abnormality was detected on radiological lung investigations in one of our patients (case 2). In contrast to peripheral sclerosing hemangioma, bronchoscopy was the main way of initially establishing the correct diagnosis of endobronchial sclerosing hemangioma in our cases. Bronchoscopy revealed a polypoid mass with a smooth and hemorrhagic surface, protruding into a proximal bronchial lumen, reminiscent of a carcinoid tumor. The endobronchial location allowed for sampling of 
the lesion during bronchoscopy. The endoscopic appearance is nonspecific, therefore, correct diagnosis requires biopsy with recognition of the characteristic histological and/or cytological features.

The cytological features that we observed from this bronchial washing are consistent with those previously reported on FNA and imprint cytology from peripheral lesions. ${ }^{3-11} \mathrm{~A}$ dual cell proliferation of round cells and pneumocytes associated with numerous foamy macrophages is the hallmark of the lesion. Round cells arranged in clusters have a characteristic cytologic appearance, with small, uniform nuclei, inconspicuous nucleoli and scant cytoplasm. In contrast to FNA descriptions, on bronchial washing the round cells largely predominate. Also, the sclerotic stromal cores and hemosiderin-laden macrophages usually described on FNA samples were not identified. This can be explained by the fact that bronchial washing is representative of cells easily detached from the tumor, while fibrotic tissue is usually cohesive and does not fall into the bronchial lumen. Also, solid component of a sclerosing hemangioma may not be sampled by a bronchial washing. The cells are more cohesive and are not easily detached from the surface. Indeed, in our second case, where the endobronchial component of the tumor was essentially solid, the bronchial washing did not reveal any tumor cells. Therefore, bronchial cytology alone may be insufficient in some cases and biopsy should be performed whenever possible.

The differential diagnosis of sclerosing hemangioma on bronchial material includes other endobronchial tumors such as adenocarcinomas either primary or metastatic, carcinoid tumors and salivary gland tumors. Although tumor cells are arranged in clusters with a papillary configuration, the absence of a necrotic background, nuclear hyperchromasia and pleomorphism, and prominent nucleoli on bronchial washing and the lack of pancytokeratin expression on bronchial biopsy help to rule out an adenocarcinoma. The past history of papillary thyroid carcinoma in our first patient, the papillary configuration of tumor cell clusters along with the presence of a few nuclear grooves and finely dispersed chromatin led to an incorrect diagnosis of metastatic papillary thyroid carcinoma. The TTF1 immunoreactivity on biopsy specimen was consistent with this diagnosis. However, nuclear molding, prominent nucleoli and ground-glass nuclei characteristic of papillary thyroid carcinomas on cytology material $^{12}$ are not seen in sclerosing hemangioma. Moreover, tumor cells did not immunostain with pancytokeratin and thyroglobulin. The cytology of carcinoid tumors demonstrates uniform, small epithelial cells with numerous bare nuclei. However, in contrast to sclerosing hemangioma, the cells have a plasmacytoid appearance, are noncohesive and isolated without clustering. ${ }^{13}$ Sclerosing hemangioma do not express neuroendocrine mar- kers. ${ }^{1}$ Although papillary in architecture, glandular papillomas are endobronchial tumors lined by columnar ciliated cells, while the epithelial lining in sclerosing hemangioma resemble pneumocytes type II. In addition, glandular papillomas lack the small, round cells seen in sclerosing hemangioma. Salivary gland tumors such as mucus gland adenomas, pleomorphic adenomas and mucoepidermoid carcinomas present as endobronchial polyps. However, they do not have the papillary pattern combined with the other characteristic solid, hemorrhagic and sclerotic patterns seen in sclerosing hemangioma. ${ }^{14}$ Mucoepidermoid carcinomas are composed of squamoid cells as well as glandular cells containing abundant mucin.

The rarity of the endobronchial location of sclerosing hemangioma may provide an insight into the question about the histogenesis of our cases. Endobronchial tumors may develop from a component of the bronchial wall, such as the bronchial epithelium in the case of papillomas or from the peribronchial glands such as the salivary gland-type tumors. In these cases, it is more likely that they arose in the peribronchial parenchyma and extended into the bronchial lumen. We did not find any immunohistochemical differences between our cases and those of peripheral sclerosing hemangioma. The morphology of the surgical resections in our cases revealed extension of the sclerosing hemangioma into the bronchial wall with the destruction of the bronchial cartilage. These observations suggest that endobronchial sclerosing hemangioma is a variant of peripheral sclerosing hemangioma, with the same histogenesis.

In conclusion, pulmonary sclerosing hemangioma may rarely present as an endobronchial mass with extension into a proximal main bronchus. Its clinical, bronchoscopic and radiological presentation differs from the usual peripheral sclerosing hemangioma. Pathologists should be aware of this rare form of presentation and should consider the possibility of a sclerosing hemangioma on bronchial washing and bronchial biopsy.

\section{Acknowledgements}

We thank Mireille Vollant for her secretarial assistance and Drs MH Koeb and M Vock-Bonnet for their help and for providing us the endobronchial biopsy material.

\section{References}

1 Devouassoux-Shisheboran M, Hayashi T, Linnoila RI, et al. A clinicopathologic study of 100 cases of pulmonary sclerosing hemangioma with immunohistochemical studies: TTF-1 is expressed in both round and surface cells, suggesting an origin from 
primitive respiratory epithelium. Am J Surg Pathol 2000;24:906-916.

2 Katzenstein AL, Gmelich JT, Carrington CB. Sclerosing hemangioma of the lung: a clinicopathologic study of 51 cases. Am J Surg Pathol 1980;4:343-356.

3 Chow LT, Chan SK, Chow WH, et al. Pulmonary sclerosing hemangioma. Report of a case with diagnosis by fine needle aspiration. Acta Cytol 1992;36:287-292.

4 Gal AA, Nassar VH, Miller JI. Cytopathologic diagnosis of pulmonary sclerosing hemangioma. Diagn Cytopathol 2002;26:163-166.

5 Gottschalk-Sabag S, Hadas-Halpern I, Glick T. Sclerosing haemangioma of lung mimicking carcinoma diagnosed by fine needle aspiration (FNA) cytology. Cytopathology 1995;6:115-120.

6 Kaw YT, Nayak RN. Fine needle aspiration biopsy cytology of sclerosing hemangioma of the lung. A case report. Acta Cytol 1993;37:933-937.

7 Krishnamurthy SC, Naresh KN, Soni M, et al. Sclerosing hemangioma of the lung: a potential source of error in fine needle aspiration cytology. Acta Cytol 1994;38: 111-112.
8 Sant F, Barcelo C, Castro P, et al. Diagnosis by fine needle aspiration cytology of sclerosing haemangioma of the lung. Cytopathology 1995;6:126-127.

9 Wang SE, Nieberg RK. Fine needle aspiration cytology of sclerosing hemangioma of the lung, a mimicker of bronchioloalveolar carcinoma. Acta Cytol 1986;30: 51-54.

10 Wojcik EM, Sneige N, Lawrence DD, et al. Fine-needle aspiration cytology of sclerosing hemangioma of the lung: case report with immunohistochemical study. Diagn Cytopathol 1993;9:304-309.

11 Iyoda A, Baba M, Saitoh $\mathrm{H}$, et al. Imprint cytologic features of pulmonary sclerosing hemangioma: comparison with well-differentiated papillary adenocarcinoma. Cancer 2002;96:146-149.

12 Kini SR, Miller JM, Hamburger JI, et al. Cytopathology of papillary carcinoma of the thyroid by fine needle aspiration. Acta Cytol 1980;24:511-521.

13 Anderson C, Ludwig ME, O’Donnell M, et al. Fine needle aspiration cytology of pulmonary carcinoid tumors. Acta Cytol 1990;34:505-510.

14 Moran CA. Primary salivary gland-type tumors of the lung. Semin Diagn Pathol 1995;12:106-122. 